\title{
INVARIANT MEASURES FOR MULTIPARAMETER DIAGONALIZABLE ALGEBRAIC ACTIONS - A SHORT SURVEY
}

\author{
ELON LINDENSTRAUSS
}

\section{Classifying measures on the one-torus}

One of the simplest dynamical systems is the map

$$
\times_{n}: x \mapsto n x \bmod 1
$$

on the unit interval, where $n$ is any natural number. In order to make this map continuous, we think of it as a map on the 1 -torus $\mathbb{T}=$ $\mathbb{R} / \mathbb{Z}$. This system is very well understood, and it has many closed invariant sets and many invariant probability measures. Indeed, let $\tau: \Sigma=\{0, \ldots, n-1\}^{\mathbb{Z}} \rightarrow \mathbb{R} / \mathbb{Z}$ be the map $\tau\left(a_{1}, a_{2}, \ldots\right)=\sum_{i=1}^{\infty} n^{-i} a_{i}$. Then any shift invariant probability measure $\nu$ on $\Sigma$, for example i.i.d. Bernoulli measure, gives rise to the $\times_{n}$-invariant measure $\mu=\tau_{*} \nu$ (and similarly for sets). Every $\times_{n}$ invariant probability measure on $\mathbb{R} / \mathbb{Z}$ is of this form, and moreover for measures $\mu$ for which $\mu(\{0\})=0$ the map $\tau_{*}$ is also one-to-one.

However, $\mathbb{R} / \mathbb{Z}$ has additional structure: it is an abelian group, and for a fixed $n$, the map $\times_{n}$ is just one out of many endomorphisms of this group. In 1967, Hillel Furstenberg considered the joint action of two such endomorphisms $\times_{n}$ and $\times_{m}$ for $n$ and $m$ multiplicatively independent (i.e., not powers of the same integer) ${ }^{1}$. This $\mathbb{Z}_{+}^{2}$ action turns out to be much more subtle. In his landmark paper [8] Furstenberg introduced the notion of disjointness in dynamical systems and ergodic theory, a notion which has proven quite central in the modem theory of these subjects, and also proves as a byproduct that the closed subsets $C \subset \mathbb{R} / \mathbb{Z}$ satisfying $\times_{n}(C) \subset C$ and $\times_{m}(C) \subset C$ are either $\mathbb{R} / \mathbb{Z}$ or finite sets of rationals.

The analogous question for measures has also been posed by Furstenberg (though apparently not in writing) in 1967, namely classifying

Date: December 28, 2004.

The author is supported by a Clay Research Fellowship; partial support was also received from NSF grant DMS-0434403.

${ }^{1}$ One can also study the interplay between $\times_{n}$ for only one $n$ and the additive structure on $\mathbb{R} / \mathbb{Z}$; this also leads to interesting questions. For more details, see [21]. 
the probability measures on $\mathbb{R} / \mathbb{Z}$ invariant under $\times_{n}$ and $\times_{m}$. This has proven substantially more difficult to resolve than the topological question. Furstenberg conjectured that any such invariant measure is a linear combination of Lebesgue measure and atomic measures supported on finite orbits of the semigroup $\left\{\times_{n^{l} m^{k}}\right\}$.

To date the best result towards Furstenberg's conjecture is due to Daniel Rudolph [37] and Aimee Johnson [10] which have shown that any measure $\mu$ which is invariant under $\times_{n}$ and $\times_{m}$ is a linear combination of Lebesgue measure and measures which have zero entropy with respect to the map $\times_{n}$; the first substantial result towards Furstenberg's conjecture, which is weaker than the Rudolph-Johnson theorem is due to Russell Lyons [25]. This is completely equivalent to the statement that the only $\times_{n}, \times_{m}$-ergodic and invariant measure on $\mathbb{R} / \mathbb{Z}$ with the entropy $h_{\mu}\left(\times_{n}\right)>0$ is Lebesgue measure. For $n, m$ relatively prime, Bernard Host [9] has given a proof of a sharper version of Rudolph theorem, which also has advantage that it is more easily quantifiable (for the extension to more general $n, m$ see [16]; see also [21]).

The $\times_{n}, \times_{m}$ action on $\mathbb{R} / \mathbb{Z}$ is prototypical for a much larger class of algebraic multiparameter actions, and these actions occur naturally in many contexts. We limit ourselves in the remainder of this note exclusively to the case of $\mathbb{R}^{k}$-actions on the locally homogeneous spaces with $k \geq 2$. This does not cover many interesting and important cases, such as actions on tori, and actions on totally disconnected groups. I also do not cover my own work on arithmetic quantum unique ergodicity, which is closely related to the topics I survey here; the interested reader can consult [17] or the expository papers $[20,19]$.

\section{More general algebraic aCtions}

The algebraic action we will consider here are by affine transformations on $\Gamma \backslash G / K$ where $G$ is a locally compact group (usually a linear group), $\Gamma<G$ a discrete subgroup, and $K<G$ compact, where by affine transformation we mean a map of the form

$$
\Gamma g K \mapsto \Gamma \Phi(g) h K
$$

with $\Phi$ an endomorphism of $G$ and $h \in G$; to make this well-defined, we need to assume that $\Phi(\Gamma) \subset \Gamma, \Phi(K) \subset K$ and $h \in G$ commutes with every $k \in K$ i.e. in the centralizer $C_{G}(K)$.

There is little loss of generality in specializing to the special case where this endomorphism is the identity, i.e. looking at the action of a closed subgroup $H<G$ by right translations on $\Gamma \backslash G / K$ where $K \subset C_{G}(H)$ is as before compact. Even Furstenberg's $\times_{n}, \times_{m}$ conjecture can be presented in this way for a suitable $G$. Also, while there 
certainly are interesting issues arising in the study of more general (even abelian!) groups $G$ such as those considered in [38], we will consider only $S$-algebraic goups $G$ - i.e. groups $G$ which are the product of finitely many linear algebraic groups over local fields of characteristic 0 (without loss of generality, $\mathbb{R}$ or $\mathbb{Q}_{p}$ for some prime $p$ ).

Now if $H$ is generated by unipotent one parameter elements (or even just by unipotents) many of the dynamical properties of this action are well understood. In the late 1980's, Gregory Margulis solved the longstanding Oppenheim conjecture about nonrational indefinite quadratic forms in three or more variables by classifying the closed $H=\mathrm{SO}(2,1)$ invariant subsets in $\mathrm{SL}(3, \mathbb{Z}) \backslash \mathrm{SL}(3, \mathbb{R})$ (see [2] for a very accessible treatment). Marina Ratner completely classified the $H$ invariant measures for any subgroup generated by unipotent one parameter elements (this has been proved in a series of paper culminating in [33]), and used this to classify orbit closures of such $H$, indeed even the behavior of single orbits [34]. Ratner's theorem, which has been extended to the $S$-arithmetic context (there are two treatments: one by Ratner [35] and one by Margulis and George Tomanov [27]), has found many diverse applications.

This, however, does not cover e.g. the case of $H$ a commutative diagonalizable group. Indeed, it seems that in view of Ratner's theorem, understanding the action of commutative groups (e.g. their invariant measures) is probably the main missing step in understanding actions of general closed, connected $H$ (see $[15$, Sec. 4b]).

Following is a prominent example: for $n \geq 2$, let $X_{n}$ be the space $\operatorname{SL}(n, \mathbb{Z}) \backslash \operatorname{SL}(n, \mathbb{R})$. We can identify $X_{n}$ with the space of lattices in $\mathbb{R}^{n}$ of covolume one by assigning to every $\operatorname{SL}(n, \mathbb{Z}) g$ the lattice $\mathbb{Z}^{n} g<\mathbb{R}^{n}$. The space $X_{n}$ is not compact: a sequence of lattices $x_{i}$ is bounded in $X_{n}$ if and only if there is some $\delta>0$ so that every vector $v_{i}$ in the lattice $x_{i}$ has size $\geq \delta$. Now take $H$ to be the group of diagonal $n \times n$ matrices of determinant 1 .

There is a sharp dichotomy between $n=2$ and $n \geq 3$. If $n=2$ then $X_{2}$ is isomorphic to a double cover of the unit cotangent bundle of the finite volume surface $\mathrm{SL}(2, \mathbb{Z}) \backslash \mathbb{H}$. Under this isomorphism, the action of $H$ becomes the geodesic flow on $\mathrm{SL}(2, \mathbb{Z}) \backslash \mathbb{H}$. As is well-known this flow is a prototypical hyperbolic flow, and has good symbolic codings which were already pioneered by Koebe, Morse and Hedlund in the beginning of the 20th-century. This is closely analogous to the situation 
with the $\times_{3}$ map acting on $\mathbb{R} / \mathbb{Z}$ which has good symbolic codings ${ }^{2}$. And using these symbolic codings, one can construct lots of invariant measures and lots of invariant sets with various properties, for example $H$-closed invariant sets which contains no periodic points and have positive fractional Hausdorff dimension.

For $n \geq 3$ the situation is drastically different. There, the dynamics of the diagonal group $H$ is much more rigid. For example, in [28] Margulis made the following conjectures regarding this action:

Conjecture 1 (Margulis). Let $X_{n}=\operatorname{SL}(n, \mathbb{Z}) \backslash \mathrm{SL}(n, \mathbb{R})$ and $H<$ $\mathrm{SL}(n, \mathbb{R})$ as above, with $n \geq 3$

(1) any bounded $H$ orbit is in fact a compact orbit.

(2) any $H$-invariant probability measure $\mu$ on $X_{n}$ is a linear combination of algebraic measures (i.e. L-invariant probability measures on closed orbit of a closed subgroup $L<\mathrm{SL}(n, \mathbb{R})$ containing $H)^{3}$.

Similar conjectures have also been made by Furstenberg (which prompted work of Shahar Mozes towards the topological question [29, 30]) as well as Anatole Katok and Ralf Spatzier [13] (who also made the first substantial progress towards classifying such invariant measures in the context of homogeneous spaces).

Note that there is a subtlety here which was not present in the cases covered by Ratner's theorem. There is no one parameter subgroup of $H$ whose action is in any way rigid: only the action of the full group $H$ (or at least a two-dimensional subgroup of this group) is rigid. This can be used to construct non-algebraic orbit closures or invariant probability measures, even for $H$ the group of $n \times n$ diagonal matrices and $G=\operatorname{SL}(n, \mathbb{R})$ on quotients $\Gamma \backslash G$ for certain lattices $\Gamma<G$, where one can create situations where the action essentially degenerates to the action of a one parameter subgroup. Because of certain coincidences, this does not happen on $X_{n}=\operatorname{SL}(n, \mathbb{Z}) \backslash \mathrm{SL}(n, \mathbb{R})$, but even in $X_{n}$ one can easily construct non-algebraic $H$-invariant and ergodic Radon measures using the same idea. This complication has been pointed out by M. Rees In the unpublished [36] and independently by Mozes in [30]; a nice account of the Rees example and some generalizations can be found in [4, Section 9].

\footnotetext{
${ }^{2}$ Though there is one important difference: for $\mathrm{SL}(2, \mathbb{Z}) \backslash \mathrm{SL}(2, \mathbb{R})$ we are considering symbolic codings for a flow and not for a single transformation which is somewhat more complicated.

${ }^{3}$ this part of the conjecture is not stated explicitly there, but follows from $[28$, Conjecture 2].
} 
As mentioned before, Margulis proved the long-standing Oppenheim conjecture by classifying orbit closures of the group $S O(2,1)$ in $X_{3}$. Similarly, even the simplest(?) $n=3$ case of either (1) or (2) in Conjecture 1 will give a proof of the following conjecture of Littlewood posed roughly at the same time as Oppenheim's conjecture (and much more):

Conjecture 2 (Littlewood (c. 1930)). Let $\|x\|$ denote the distance from $x \in \mathbb{R}$ to the closest integer. Then

$$
\varliminf_{n \rightarrow \infty} n\|n \alpha\|\|n \beta\|=0
$$

for any real numbers $\alpha$ and $\beta$.

This implication has been discovered in a different terminology long before Furstenberg's pioneering work regarding the rigidity of multiparameter actions (and long before Margulis' proof of the Oppenheim conjecture using dynamical techniques) by J. W. S. Cassels and H. P. F. Swinnerton-Dyer [1]; however, it was Margulis who first recast this in dynamical terms [26].

Unlike the case of the one torus, there is no reason to believe that in the context of actions on locally homogeneous spaces the topological question, e.g. (1) of Conjecture 1, is substantially easier then the measure theoretic question (e.g. (2) of the same question). It is, however, true that using [1] or [24] one can deduce (1) of Conjecture 1 from (2).

\section{Partial Results towards Classifying invariant Measures IN THE LOCALLY HOMOGENEOUS CASE}

In $[13,14]$, Katok and Spatzier made the first steps towards classifying invariant measures in the locally homogeneous case, in particular covering the case of the diagonal group (which will denote as before by $H$ ) acting on $X_{n}$. Their work contains some elements which are geometric analogous to the techniques used by Rudolph for the onedimensional torus in [37], as well as some additional new ingredients, particularly in handling actions of abelian groups with nontrivial Jordan form. A good exposition of their method (though without mention of the locally homogeneous case), clarifying some aspects of the original work, can be found in [11]. Later, Boris Kalinin and Spatzier further developed this method [12], with nice ergodic theoretic applications we will discuss in the next section.

For classifying measures, not all elements of $H$ are created equal. The most important one parameter subgroups are those nontrivial elements $\operatorname{diag}\left(h_{1}, h_{2}, \ldots, h_{n}\right) \in H$ which have at least two entries which are 
equal, say $h_{i}=h_{j}$. Such elements act isometrically, indeed only by translations, on the leaves of the $H$-invariant foliation of $X_{n}$ into orbits of the unipotent group

$U_{i j}=\left\{\left(u_{k l}\right)_{k l}: u_{k k}=1\right.$ for all $k, u_{k l}=0$ for all $k \neq l$ except $\left.(k, l)=(i, j)\right\}$, and there is a substantial amount of information which can be learned about the measure $\mu$ merely because it is invariant under even a single element with this partial isometric property. Implicitly, this partial isometric feature of the action of some elements of the acting group is used already in [37].

For $X_{n}$ the techniques of Katok and Spatzier (as well as the later work of Kalinin and Spatzier) give that if the subgroups

$$
H^{i j}=\left\{\operatorname{diag}\left(h_{1}, h_{2}, \ldots, h_{n}\right): h_{i}=h_{j}\right\}
$$

act ergodically ${ }^{4}$ with respect to $\mu$, and if there is any one parameter subgroup of $H$ which acts with positive entropy then $\mu$ needs to be algebraic.

Of these two assumptions, the assumption regarding ergodicity of the groups $H^{i j}$ (which did not appear in Rudolph's theorem) is the more restrictive one. The reason for this is that in a typical application of measure classification results such as Ratner's theorems or the conjectured measure classification for the diagonal flow in Conjecture 1 the measure one analyzes is obtained as a weak* limit of measures on which we have some control (for example, empirical measure on orbit segments). Ergodicity properties are not stable under weak* limits. Entropy, on the other hand, is well behaved under weak* limits: though this is not true in the greatest generality, in the type of systems we consider entropy is an upper semicontinuous function of the measure with respect to the weak* topology.

In the last couple of years, and there has been substantial progress towards eliminating the need for an ergodicity assumption in the locally homogeneous context, and research in this direction is still in progress. There are two complementary ways of proving such results: one of them developed by Manfred Einsiedler and Katok in [4] uses non-commutativity of invariant contracting foliations, such as the foliation by orbits of $U_{i j}$ and $U_{j k}$ for $X_{n}$ togather with a simple but very useful lemma regarding a product structue of some conditional measures under the multiparameter diagonalizable flows discussed here.

\footnotetext{
${ }^{4}$ The assumptions that $H^{i j}$ act ergodically can be weakened somewhat, and the analogous weaker statment is very important in some special cases, such as for the $\times n, \times m$-action on $\mathbb{R} / \mathbb{Z}$ or for $\mathbb{Z}^{k-1}$ actions on $k$-dimensional tori, but it does not seem to be very useful in the locally homogeneous context.
} 
In particular, Einsiedler and Katok have proved that any $H$ invariant measure in $X_{n}$ which has positive entropy under all elements of the diagonal group is algebraic - in fact, under this assumption the measure needs to be Haar measure on $X_{n}$. Einsiedler and Katok have generalized their results to a very general class of groups (the original paper covered only $\mathbb{R}$-split groups) in [5].

This approach cannot be used for every $G$. For example, for the action of the two-dimensional diagonal group on $\Gamma \backslash \mathrm{SL}(2, \mathbb{R}) \times \mathrm{SL}(2, \mathbb{R})$ there are no non-commuting invariant contracting foliations. There a completely different method needs to be used: one that uses some of the ideas and techniques in Ratner's work on the rigidity of unipotent flows, particularly from her earlier works [31, 32]. In [17], we show that a measure on $\Gamma \backslash \mathrm{SL}(2, \mathbb{R}) \times \mathrm{SL}(2, \mathbb{R})$ invariant and ergodic under the action of the two-dimensional diagonal group (in fact it is enough that it be invariant under the action of the diagonal group in one $\operatorname{SL}(2, \mathbb{R})$ factor, and recurrent under the action of the other $\operatorname{SL}(2, \mathbb{R})$ factor, which is a substantially weaker assumption - see [17] for exact statement) and which has positive entropy with respect to some one parameter diagonal subgroup, is algebraic (again, in this case, Haar measure on the quotient).

Combining these two techniques, one has the following results for $X_{n}$ :

Theorem 3.1 ( Einsiedler, Katok and L. [6]). Let $\mu$ be a $H$-invariant and ergodic measure on $X_{n}$. Assume that there is some one parameter subgroup of $H$ with respect to which $\mu$ has positive measure. Then $\mu$ is algebraic, and is not compactly supported. If $n$ is prime then $\mu$ is the Haar measure on $X_{n}$.

This theorem, precisely because of the good behavior of entropy with respect to weak* limits, implies the following partial result towards Littlewood Conjecture:

Theorem 3.2 ( Einsiedler, Katok and L. [6]). The set of $(\alpha, \beta) \in \mathbb{R}^{2}$ for which $\underline{\lim }_{n \rightarrow \infty} n\|n \alpha\|\|n \beta\|>0$ has Hausdorff dimension zero.

As mentioned above, a key role in the proof of these measure classification results play the singular direction in which the action is partially isometric. Understanding such actions for their own sake seems to be a fruitful direction of research; see [22, 23, 18] for more details; the results of [17] are also best seen in this light. 


\section{JOINING AND ISOMORPHISM RIGIDITY OF DIAGONALIZABLE ACTIONS ON LOCALLY HOMOGENEOUS SPACES ${ }^{5}$}

Earlier we have mentioned the dichotomy between the action of the diagonal group on $X_{2}$ and the action of the corresponding group in $X_{n}$ for $n \geq 3$. In this section, we give another facet of this dichotomy.

We start with generalities: let $H$ be some group, and suppose this group $H$ acts on the two spaces $X$ and $X^{\prime}$. Let $m, m^{\prime}$ be $H$ invariant measures on $X$ and $X^{\prime}$ respectively. A joining of $(X, H, m)$ and $\left(X^{\prime}, H, m^{\prime}\right)$ is a measure on $X$ and $X^{\prime}$ invariant under the diagonal action of $H$ on $X \times X^{\prime}$ whose push forward under the projection to $X$ $\left(X^{\prime}\right)$ is $m$ ( $m^{\prime}$ respectively). Any measurable isomorphism $\phi$ between $(X, m)$ and $\left(X^{\prime}, m^{\prime}\right)$ commuting with the $H$ action gives rise to a joining between $(X, H, m)$ and $\left(X^{\prime}, H, m^{\prime}\right)$ supported on the graph of this isomorphism.

For $n=2$ it is possible to construct non-algebraic joinings of $\left(X_{2}, H, m\right)$ with itself or other one parameter flows, and a similar statement should be true for isomorphisms. This is in stark contrast with what happens for $n \geq 3$. Kalinin and Spatzier [12] proved a general isomorphism rigidity theorem for such multidimensional actions; in particular they proved:

Theorem 4.1 (Kalinin and Spatzier [12]). Let $G_{1}, G_{2}$ be connected semisimple Lie groups without compact factors. For $i=1,2$, let $\Gamma_{i}<$ $G_{i}$ be a uniform lattice, $m_{i}$ Haar measure on $\Gamma_{i} \backslash G_{i}$ and let $\rho_{i}$ be an embedding of $\mathbb{R}^{k}(k \geq 2)$ to the Cartan subgroup of $G_{i}$. Then any measurable isomorphism between the $\mathbb{R}^{k}$ actions corresponding to $\rho_{i}$ on $\left(\Gamma_{i} \backslash G_{i}, m_{i}\right)$ is algebraic.

The assumption that the lattices $\Gamma_{i}$ are uniform does not seem to be essential for the proof.

More generally, one can consider joinings of such actions. Because isomorphisms gives rise to particular kind of joinings, this is a more general question, and as we shall see below it has applications to equidistribution. Kalinin and Spatzier give some results toward classifying joinings, but because they rely on the technology of [13] they need to assume ergodicity of the joining with respect to one parameter subgroups. Using the results of Einsiedler and Katok in [5], and some ideas of Einsiedler and Tom Ward from [3] jointly with Einsiedler we have the following:

\footnotetext{
${ }^{5}$ Again we limit ourselves to flows on locally homogeneous spaces; a lot of interesting work has been done in other contexts by Einsiedler, Katok, Kalinin, Schmidt, Thouvenot, Ward and many others.
} 
Theorem 4.2 (Einsiedler-L. [7]). Let $G_{1}, G_{2}$ be connected semisimple Lie groups, $\Gamma_{i}<G_{i}$ a lattice, $m_{i}$ Haar measure on $\Gamma_{i} \backslash G_{i}$, and $\rho_{i}$ be an embedding of $\mathbb{R}^{k}(k \geq 2)$ to the Cartan subgroup of $G_{i}$ such that the image of $\rho_{i}\left(\mathbb{R}^{k}\right)$ on every factor of $G_{i}$ has dimension $\geq 2$. Then any ergodic joining between the $\mathbb{R}^{k}$ actions corresponding to $\rho_{i}$ on $\left(\Gamma_{i} \backslash G_{i}, m_{i}\right)$ is algebraic.

In particular, any self joining of $X_{n}$ with itself for $n \geq 3$ is algebraic. Theorem 4.2 can be used for example to show the following:

Theorem 4.3 (Einsiedler-L. [7]). Let $G$ be a connected simple Lie group, of $\mathbb{R}$ rank $\geq 2$. Let $\Gamma_{1}, \Gamma_{2}$ be two lattices which cannot be conjugated so as to be commensurable. Suppose $x_{1} \in \Gamma_{1} \backslash G$ and $x_{2} \in \Gamma_{2} \backslash G$ have the property that their orbit under the $\mathbb{R}$-Cartan subgroup $H<G$ is equidistributed ${ }^{6}$. Then the same holds for the orbit of $\left(x_{1}, x_{2}\right)$ in $\Gamma_{1} \backslash G \times \Gamma_{2} \backslash G$ under the diagonal embedding of $H$.

\section{ACKNOWLeDGments}

I would like to thank the organizing committee of the 4ECM for giving me the opportunity to present my work both orally and in writing to a wide mathematical community, as well as Ari Laptev for his patience.

\section{REFERENCES}

[1] J. W. S. Cassels and H. P. F. Swinnerton-Dyer. On the product of three homogeneous linear forms and the indefinite ternary quadratic forms. Philos. Trans. Roy. Soc. London. Ser. A., 248:73-96, 1955.

[2] S. G. Dani and G. A. Margulis. Values of quadratic forms at integral points: an elementary approach. Enseign. Math. (2), 36(1-2):143-174, 1990.

[3] M. Einsiedler and T. Ward. Entropy geometry and disjointness for zerodimensional algebraic actions. preprint, 21 pages.

[4] Manfred Einsiedler and Anatole Katok. Invariant measures on $G / \Gamma$ for split simple Lie groups G. Comm. Pure Appl. Math., 56(8):1184-1221, 2003. Dedicated to the memory of Jürgen K. Moser.

[5] Manfred Einsiedler and Anatole Katok. Rigidity of measures - the high entropy case, and non-commuting foliations. preprint, 54 pages, 2004.

[6] Manfred Einsiedler, Anatole Katok, and Elon Lindenstrauss. Invariant measures and the set of exceptions to littlewoods conjecture. to appear Annals of Math. (45 pages), 2004.

\footnotetext{
${ }^{6}$ more precisely, let $\nu_{r}$ be the uniform measure on the ball of radius $r$ in $H$ centered at the identity (with respect to e.g. a left $G$-invariant Riemannian metric on $G$, bi-invariant under its maximal compact subgroup). Then the push forward of $\nu_{r}$ to $\Gamma_{i} \backslash G$ under the map $g \mapsto x_{i} . g^{-1}$ tends as $r \rightarrow \infty$ in the weak* topology to the Haar measure.
} 
[7] Manfred Einsiedler and Elon Lindenstrauss. Joining of semisimple actions on locally homogeneous spaces. in preparation, 2004.

[8] Harry Furstenberg. Disjointness in ergodic theory, minimal sets, and a problem in Diophantine approximation. Math. Systems Theory, 1:1-49, 1967.

[9] Bernard Host. Nombres normaux, entropie, translations. Israel J. Math., 91(13):419-428, 1995.

[10] Aimee S. A. Johnson. Measures on the circle invariant under multiplication by a nonlacunary subsemigroup of the integers. Israel J. Math., 77(1-2):211-240, 1992.

[11] Boris Kalinin and Anatole Katok. Invariant measures for actions of higher rank abelian groups. In Smooth ergodic theory and its applications (Seattle, WA, 1999), volume 69 of Proc. Sympos. Pure Math., pages 593-637. Amer. Math. Soc., Providence, RI, 2001.

[12] Boris Kalinin and Ralf Spatzier. Rigidity of the measurable structure for algebraic actions of higher rank abelian groups. to appear in Ergodic Theory Dynam. Systems.

[13] A. Katok and R. J. Spatzier. Invariant measures for higher-rank hyperbolic abelian actions. Ergodic Theory Dynam. Systems, 16(4):751-778, 1996.

[14] A. Katok and R. J. Spatzier. Corrections to: "Invariant measures for higherrank hyperbolic abelian actions" [Ergodic Theory Dynam. Systems 16 (1996), no. 4, 751-778; MR 97d:58116]. Ergodic Theory Dynam. Systems, 18(2):503$507,1998$.

[15] Dmitry Kleinbock, Nimish Shah, and Alexander Starkov. Dynamics of subgroup actions on homogeneous spaces of Lie groups and applications to number theory. In Handbook of dynamical systems, Vol. 1A, pages 813-930. NorthHolland, Amsterdam, 2002.

[16] Elon Lindenstrauss. p-adic foliation and equidistribution. Israel J. Math., 122:29-42, 2001.

[17] Elon Lindenstrauss. Invariant measures and arithmetic quantum unique ergodicity. to appear in Annals of Math. (54 pages), 2003.

[18] Elon Lindenstrauss. Recurrent measures and measure rigidity. To appear in the proceeding of the II Workshop on Dynamics and Randomness, Santiago de Chile, Dec. 9-13, 2002. Eds. A. Maass, S. Martinez, J. San Martin. (25 pages), 2003.

[19] Elon Lindenstrauss. Rigidity of multiparameter actions. submitted to the forthcoming volume(s) of the Israel J. of Math. dedicated to H. Furstenberg (26 pages), 2003.

[20] Elon Lindenstrauss. Arithmetic quantum unique ergodicity and adelic dynamics. preprint (24 pages), 2004.

[21] Elon Lindenstrauss, David Meiri, and Yuval Peres. Entropy of convolutions on the circle. Ann. of Math. (2), 149(3):871-904, 1999.

[22] Elon Lindenstrauss and Klaus Schmidt. Invariant measures of nonexpansive group automorphisms. to appear Israel J. Math. (28 pages), 2003.

[23] Elon Lindenstrauss and Klaus Schmidt. Symbolic representations of nonexpansive group automorphisms. to appear in Israel J. Math (34 pages), 2003.

[24] Elon Lindenstrauss and Barak Weiss. On sets invariant under the action of the diagonal group. Ergodic Theory Dynam. Systems, 21(5):1481-1500, 2001. 
[25] Russell Lyons. On measures simultaneously 2- and 3-invariant. Israel J. Math., 61(2):219-224, 1988.

[26] G. A. Margulis. Oppenheim conjecture. In Fields Medallists' lectures, volume 5 of World Sci. Ser. 20th Century Math., pages 272-327. World Sci. Publishing, River Edge, NJ, 1997.

[27] G. A. Margulis and G. M. Tomanov. Invariant measures for actions of unipotent groups over local fields on homogeneous spaces. Invent. Math., 116(1-3):347392, 1994.

[28] Gregory Margulis. Problems and conjectures in rigidity theory. In Mathematics: frontiers and perspectives, pages 161-174. Amer. Math. Soc., Providence, RI, 2000.

[29] Shahar Mozes. On closures of orbits and arithmetic of quaternions. Israel J. Math., 86(1-3):195-209, 1994.

[30] Shahar Mozes. Actions of Cartan subgroups. Israel J. Math., 90(1-3):253-294, 1995.

[31] Marina Ratner. Factors of horocycle flows. Ergodic Theory Dynam. Systems, 2(3-4):465-489, 1982.

[32] Marina Ratner. Horocycle flows, joinings and rigidity of products. Ann. of Math. (2), 118(2):277-313, 1983.

[33] Marina Ratner. On Raghunathan's measure conjecture. Ann. of Math. (2), 134(3):545-607, 1991.

[34] Marina Ratner. Raghunathan's topological conjecture and distributions of unipotent flows. Duke Math. J., 63(1):235-280, 1991.

[35] Marina Ratner. Raghunathan's conjectures for Cartesian products of real and p-adic Lie groups. Duke Math. J., 77(2):275-382, 1995.

[36] M. Rees. Some $R^{2}$-anosov flows. 1982.

[37] Daniel J. Rudolph. $\times 2$ and $\times 3$ invariant measures and entropy. Ergodic Theory Dynam. Systems, 10(2):395-406, 1990.

[38] Klaus Schmidt. Dynamical systems of algebraic origin, volume 128 of Progress in Mathematics. Birkhäuser Verlag, Basel, 1995. 